\title{
OBSERVACIONES ACERCA DE ALGUNOS TUBIFICIDAE (OLIGOCHAETA) DE LA PENINSULA IBÉRICA, CON LA DESCRIPCION DE PHALLODRILLUS RIPARIUS N. SP.
}

\author{
por N. GIANI ${ }^{1}$ y E. MARTINEZ-ANSEMIL²
}

\begin{abstract}
Se describe una nueva especie de Tubificidae, Phallodrilus riparius $\mathrm{n}$. sp., capturada en el río Tambre (Galicia). La forma y el número de sedas peniales, la forma de las espermatecas y la presencia de tubo digestivo son los principales caracteres que, combinados, la distinguen de los demás representantes del género. Contrariamente a la inmensa mayoría de los Phallodrilus conocidos, $P$. riparius n. sp. es una especie de agua dulce.

Se observa y estudia la variación con la edad del binomio sedas ventrales bífidas-sedas ventrales con punta simple en una población de Peloscolex velutinus (Grube) y se describen, finalmente, las sedas genitales de Rhyacodrilus subterra. neus Hrabe y una forma original de Tubifícido (Tubificidae gen. sp.) que no hemos podido encontrar hasta Ia fecha en estado de madurez sexual.
\end{abstract}

\section{Observations sur quelques Tubificidae (Oligochaeta) de la Péninsule lbérique, avec la description de Phallodrilus riparius n. sp.}

Nous décrivons une nouvelle espèce de Tubificidae, Phallodrilus riparius $\mathrm{n}$. $\mathrm{sp}$., récoltée dans la rivière Tambre (Galice). La forme et le nombre de soies péniennes, la forme des spermathèques et la présence d'un tube digestif sont les principaux caractères qui, combinés, la distinguent des autres représentants du genre. Contrairement à la grande majorité des Phallodrilus connus, Phallodrillus riparius $\mathrm{n}$. sp. est une forme d'eau douce.

Nous étudions la variation avec l'âge du rapport entre le nombre de soies ventrales bifides et à pointe simple chez Peloscolex velutinus (Grube) et nous décrivons, finalement, les soies génitales de Rhyacodrilus subterraneus Hrabe et ine forme originale de Tubificidé (Tubificidae gen. sp.) que nous n'avons pas pu rencontrer, jusqu'à présent, à l'état de maturité sexuelle.

\section{Observations on several tubificids (Oligochaeta) from the Iberian peninsula, with a description of Phallodrilus riparius $\mathbf{n}$. sp.}

We have described a new species of Tubificidae, Phallodrilus riparius n. sp., collected from the river Tambre (Galice). The form and number of the penial chaetae, the form of the spermathecae and the presence of a digestive tube are the principal characters which, in combination, are used to separate this species from others in the same genus. In contrast to most Phallodrilus spp., P. riparius n. sp. is a freshwater form.

1. Laboratoire d'Hydrobiologie, Université Paul-Sabatier, 118, route de Narbonne, 31062 Toulouse Cedex.

2. Colegio Universitario de Orense, C. General Franco, 35 - Orense (Espagne). 
We have studied the variation with age of the ratio of ventral bifid chaetae and those that are simple pointed in Peloscolex velutinus (Grube). Finally, we have described the genital chaetae of Rhyacodrilus subterraneus Hrabe and an original form of tubificid (Tubificidae gen. sp.) that we have not been able to find, up to present, in the sexually mature stage.

En el transcurso de un estudio sobre los Oligoquetos del río Tambre - Galicia - (Martinez-Ansemil, 1981), hemos capturado una nueva especie de Phallodrilus (Tubificidae). Su descripción, junto con diversas observaciones sobre la morfología de Rhyacodrilus subterraneus Hrabe y Peloscolex velutinus (Grube), tendentes a completar su diagnosis, y la descripción de una forma de Tubificidae que, desafortunadamente no hemos podido encontrar todavía en estado de madurez sexual, constituyen el objetivo del presente trabajo.

Todas nuestras observaciones fueron efectuados a partir de especimenes procedentes del río Tambre, previamente fijados en formol al $4 \%$. Las principales caracteríisticas de las estaciones de captura han sido ya señalades en dos trabajos precedentes (Martinez-Ansemil y Giani, 1980 ; Martinez-Ansemil, 1981).

\section{1. - PHALLODRILUS RIPARIUS N. SP.}

\subsection{Material examinado — hábitat}

Hemos capturado un total de 9 individuos de esta nueva especie : 3 en Ponte Carreira (Lat. $N=43^{\circ} 1^{\prime} 20^{\prime \prime}$, Long. $W=4^{\circ} 33^{\prime} 50^{\prime \prime}$ ), 4 en Sigüeiro (Lat. $\mathrm{N}=42^{\circ} 57^{\prime} 50^{\prime \prime}$, Long. $\mathrm{W}=4^{\circ} 45^{\prime} 30^{\prime \prime}$ ) y 2 en Ponte Maceira (Lat. $\mathrm{N}=42^{\circ} 54^{\prime} 10^{\prime \prime}$, Long. $\mathrm{W}=5^{\circ} 0^{\prime} 40^{\prime \prime}$ ) ; estas estaciones se encuentran a 100,70 y $26 \mathrm{~km}$. respectivamente de la desembocadura del río. Todos Ios especímenes se hallaban presentes en fondos de cantos rodados bajo una corriente lenta, moderada o rápida; de ellos, 7 , se encontraban en estado de madurez sexual (1 el 31-3-79, 3 el 10-6-79, 1 el (-10-79 y 2 el 4-7-81).

\subsection{Diagnosis}

\subsubsection{Caracteres EXTERnos}

29 segmentos y $3,2 \mathrm{~mm}$. de longitud (único ejemplar entero, fijado en formol). De 60 a $70 \mu$ de diámetro en la región preclitelar y de 150 a $160 \mu$ a nivel del clitelo (medidas correspondientes a individuos ligeramente comprimidos).

Prostomio redondeado (fig. 1D) y ligeramente más ancho ( 90 a $95 \mu$ ) que largo $(75$ a $90 \mu)$. 

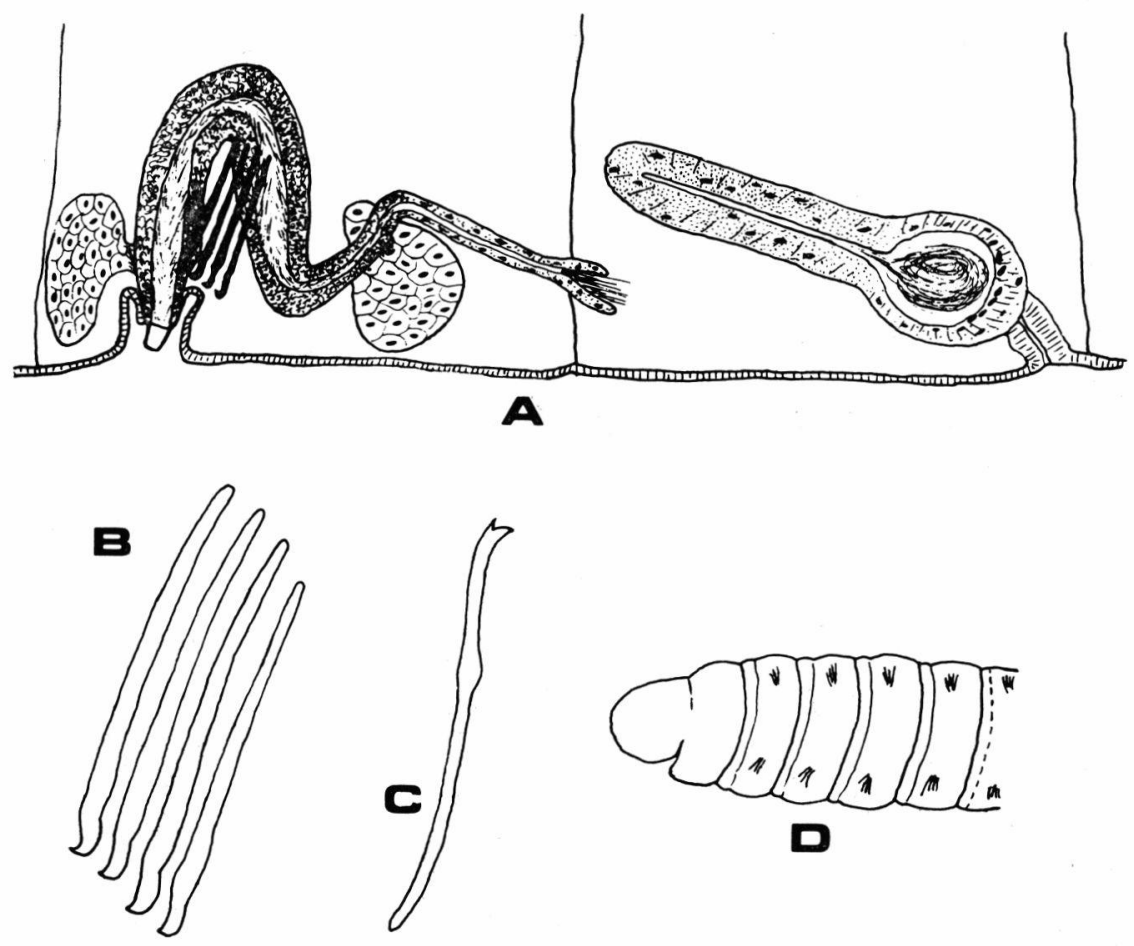

FIG. 1. - Phallodrilus riparius n. sp.: A. aparato genital; B. sedas peniales;

C. sedas somáticas; D. región anterior.

Tegumento con segmentación secundaria, consistente en un sólo surco presente en la parte anterior de cada segmento. Dicho surco es particularmente neto en los segmentos II, III, IV y V (fig. 1D).

Clitelo muy prominente y desarrollado, ocupando la mitad del segmento $\mathrm{X}$ y los segmentos XI y XII en su totalidad (1/2X-XII).

Ausencia de sedas capilares. Sedas dorsales y ventrales iguales(fig. 1C). Son sedas bífidas, muy cortas (alrededor de $30 \mu$ de longitud), finas (1,1 a $1,5 \mu$ de diámetro) y con el diente superior más pequeño que el inferior; están agrupadas en haces de 2 a 5 en la región preclitelar (generalmente 3 , salvo en III, IV, V y VI, donde siempre hay 4 o 5) y de 2 o 3 (generalmente 3 ) en la región postclitelar.

Sedas espermatecales no diferenciadas. 4 sedas peniales por cada haz ventral; son sedas con punta simple, cuya longitud varía entre 33 y $36 \mu$ y cuyo espesor es de unas $2,5 \mu$ (fig. 1B).

Dos poros espermatecales y dos poros $\hat{\delta}$. Ambos desembocan en linea con las sedas ventrales, los pirmeros en el segmento $\mathrm{X}$, cerca del septo IX/X, y los segundos en la parte posterior del segmento XI. 


\subsubsection{CARACTERES INTERNOS}

Presencia de glándulas faríngeas en los metámeros IV, V y VI.

Un par de testículos en $\mathrm{X}$.

Dos atrios, alargados y curvados en forma de herradura, de 140 a $180 \mu$ de longitud y 14 a $15 \mu$ de diámetro en su parte más ancha (fig. 1A); su cavidad media es relativamente amplia y se halla tapizada por un epitelio ciliado. Cada atrio presenta un pseudopene en su parte terminal y se prolonga apicalmente en un conducto deferente cuyo diámetro varía entre 4,7 y 5,7 $\mu$ y cuya longitud es netamente inferior a la del propio atrio. Existen dos próstatas por cada atrio, una de ellas se halla situada en su parte proximal y la otra cerca de la entrada del conducto deferente.

Dos espermatecas alargadas que ocupan toda la longitud del segmento X. Cada una de ellas está formada por un conducto proximal corto y una "bombilla » distal de 65 a $85 \mu$ de longitud en la que se distinguen a su vez: una parte anterior esférica de 23 a $35 \mu$ de diámetro que contiene un amasijo de espermatozoides, y una parte posterior tubular de alrededor de $50 \mu$ de longitud y 15 a $19 \mu$ de diámetro.

\subsection{Discusión}

Por falta de especímenes desarrollados sexualmente, en buen estado de conservación y en número suficiente, en dos trabajos precedentes (Martinez-Ansemil y Giani, 1980; Martinez-Ansemil, 1981), no habíamos podido encuadrar taxonómicamente con precisión esta nueva especie de Tubificidae capturada en el Tambre; la semejanza de su morfología externa con la de los representantes del género Spiridion, nos había inducido a denominarla Spiridion ? sp. El estudio de material suplementario nos ha permitido ahora completar su diagnosis e incluirla dentro del género Phallodrilus. En efecto, la presencia de dos glándulas prostáticas por cada atrio y su posición sobre el mismo, la entrada apical de cada conducto deferente en su atrio respectivo, las sedas peniales y la forma y posición de las espermatecas de nuestros especímenes, son características de este género tal como fué redefinido por Erseus (1979 a y b).

El propio Erseus (1979 a y 1981), distribuye las especies de Phallodrillus en cuatro grupos que se diferencian entre si por la combinación de los caracteres siguientes : presencia o ausencia de tubo digestivo, presencia o ausencia de sedas espermatecales diferenciadas, presencia o ausencia y número de sedas peniales. $P$. riparius n. sp. pertenece al grupo caracterizado por la presencia de tubo digestivo, la ausencia de sedas espermatecales diferenciadas y la presencia de $4 \mathrm{o}$ más sedas peniales por cada poro y se diferencia fundamentalmente 
de las demás especies del mismo por la forma de sus espermatecas y por el número y la forma de sus sedas peniales.

Según Erseus (1981), el número de especies del género Phallodrilus conocidas hasta la fecha era de 29 . Este autor (1979 a), subraya que, de entre todas ellas, tan sólo $P$. hallae Cook y Hiltunen vive en agua dulce (Lago superior, América del Norte) y, a pesar que $P$. aquae. dulcis Hrabe fué capturada en el río Weser (Alemania), en varias estaciones escalonadas hasta $95 \mathrm{~km}$. de su desembocadura, considera esta especie como oligohalina, señalando una posible contaminación por sales de este río. A la vista de estas observaciones, resulta pues interesante destacar la presenica en Europa de la nueva especie de agua dulce $P$. riparius que nosotros hemos capturado en el Tambre, en tres estaciones situadas entre 26 y $100 \mathrm{~km}$. de su desembocadura y cuyas aguas están poco mineralizadas: conductividad $=38$ a 270 $\mu \mathrm{mhos} / \mathrm{cm} / \mathrm{cm}^{2}$ (véase Martinez-Ansemil, 1981 ).

N.B. El nombre que hemos atribuido a esta especie hace alusión al medio en el que la hemos encontrado (del latin riparia $=$ río).

\section{2. - PELOSCOLEX VELUTINUS (GRUBE, 1879)}

Basándose únicamente en la naturaleza de las sedas ventrales (con punta simple, bífidas o los dos tipos a la vez en un mismo individuo), la identidad de $P$. velutinus ha sido objeto de una amplia discusión entre diversos autores (Randolph, 1892; Piguet y Bretscher, 1913; Hrabe, 1964 ; Brinkhurst y Jamieson, 1971 ; Hrabe, 1973...). El examen de la quetotaxia de 53 de los 707 individuos de esta especie caturados a lo largo de nuestro estudio sobre los oligoquetos del río Tambre (Martinez-Ansemil, 1981), nos ha permitido constater que la relación entre el número total de sedas ventrales con punta bífida y el de sedas ventrales con punta simple varía en función de la edad de los individuos (número de segmentos), lo cual nos lleva a considerar que la naturaleza de sus sedas ventrales no puede constituir por sí sola un carácter sistemático fiable.

Nuestras observaciones (tabla I) muestran que los individuos jóvenes poseen únicamente, o casi, sedas bífidas; a medida que se van desarrollando, el número de sedas bífidas disminuye mientras que el de sedas con punta simple va en aumento y, finalmente, los individuos cuyo número de segmentos es superior a 38 , poseen ya exclusivamente, o casi, sedas con punta simple. Es muy probable que las observaciones de los autores citados anteriormente hayan sido realizadas sobre un pequeño número de individuos, por lo que no les ha sido posible detectar el mencionado cambio en la naturaleza de las sedas con la edad. 
TABLA I. - Variación de la quetotaxia de $P$. velutinus en el curso de su desarrollo $\left(\mathrm{n}^{\circ}\right.$ seg. $=$ número de segmentos, $\mathrm{n}^{\circ}$ s.b. $=$ número de sedas ventrales bífidas, $n^{\circ}$ s.s. = número de sedas ventrales con punta simple) $-\mathrm{El}$ número de sedas aqui indicado concierne un sólo lado del animal - .

\begin{tabular}{|c|c|c|c|c|c|c|c|c|c|c|c|c|c|c|}
\hline $\begin{array}{ll}\mathrm{li}^{\circ} & \text { seg. } \\
\mathrm{n}^{\mathrm{o}} & \text { s.b. } \\
\mathrm{n}^{\mathrm{o}} & \text { s.s. } \\
& \text { s.s. }\end{array}$ & $\begin{array}{r}18 \\
22 \\
0\end{array}$ & $\begin{array}{r}21 \\
25 \\
0\end{array}$ & $\begin{array}{r}22 \\
18 \\
8\end{array}$ & $\begin{array}{r}22 \\
23 \\
5\end{array}$ & $\begin{array}{r}22 \\
23 \\
5\end{array}$ & $\begin{array}{r}23 \\
21 \\
6\end{array}$ & $\begin{array}{r}24 \\
19 \\
9\end{array}$ & $\begin{array}{l}24 \\
29 \\
13\end{array}$ & $\begin{array}{r}24 \\
30 \\
0\end{array}$ & $\begin{array}{l}25 \\
19 \\
10\end{array}$ & $\begin{array}{r}25 \\
25 \\
5\end{array}$ & $\begin{array}{r}25 \\
24 \\
7\end{array}$ & $\begin{array}{r}26 \\
25 \\
8\end{array}$ & $\begin{array}{r}26 \\
22 \\
8\end{array}$ \\
\hline$\overline{\text { s.s. }+ \text { s.b. }} \times 100$ & 0 & 0 & 31 & 18 & 18 & 29 & 32 & 31 & 0 & 35 & 17 & 23 & 24 & 27 \\
\hline $\begin{array}{ll}\mathrm{g}^{\circ} & \text { seg. } \\
n^{\circ} & \text { s.b. } \\
n^{\circ} & \text { s.s. } \\
& \text { s.s. }\end{array}$ & $\begin{array}{l}29 \\
13 \\
19\end{array}$ & $\begin{array}{l}29 \\
11 \\
23\end{array}$ & $\begin{array}{l}30 \\
10 \\
25\end{array}$ & $\begin{array}{r}30 \\
0 \\
35\end{array}$ & $\begin{array}{l}30 \\
20 \\
15\end{array}$ & $\begin{array}{l}30 \\
18 \\
16\end{array}$ & $\begin{array}{r}31 \\
0 \\
35\end{array}$ & $\begin{array}{l}32 \\
21 \\
16\end{array}$ & $\begin{array}{r}32 \\
3 \\
35\end{array}$ & $\begin{array}{l}33 \\
25 \\
13\end{array}$ & $\begin{array}{l}34 \\
15 \\
25\end{array}$ & $\begin{array}{r}38 \\
0 \\
43\end{array}$ & $\begin{array}{r}38 \\
0 \\
41\end{array}$ & $\begin{array}{r}38 \\
0 \\
47\end{array}$ \\
\hline$\overline{\text { s.s. }+ \text { s.b. }} \times 100$ & 59 & 68 & 71 & 100 & 43 & 47 & 100 & 43 & 92 & 34 & 63 & 100 & 100 & 100 \\
\hline $\begin{array}{ll}\mathrm{n}^{\circ} & \text { seg. } \\
\mathrm{n}^{\circ} & \text { s.b. } \\
\mathrm{n}^{\circ} & \text { s.s. } \\
& \text { s.s. }\end{array}$ & $\begin{array}{r}38 \\
0 \\
43\end{array}$ & $\begin{array}{r}39 \\
3 \\
41\end{array}$ & $\begin{array}{r}40 \\
2 \\
42\end{array}$ & $\begin{array}{r}40 \\
1 \\
44\end{array}$ & $\begin{array}{r}40 \\
0 \\
47\end{array}$ & $\begin{array}{r}41 \\
6 \\
40\end{array}$ & $\begin{array}{l}44 \\
10 \\
39\end{array}$ & $\begin{array}{r}44 \\
0 \\
50\end{array}$ & $\begin{array}{r}47 \\
0 \\
50\end{array}$ & $\begin{array}{r}47 \\
0 \\
53\end{array}$ & $\begin{array}{r}49 \\
2 \\
51\end{array}$ & $\begin{array}{r}49 \\
0 \\
54\end{array}$ & $\begin{array}{r}51 \\
0 \\
68\end{array}$ & $\begin{array}{r}53 \\
2 \\
56\end{array}$ \\
\hline$\overline{\text { s.s }+ \text { s.b. }} \times 100$ & 100 & 93 & 95 & 98 & 100 & 71 & 80 & 100 & 100 & 100 & 96 & 100 & 100 & 97 \\
\hline $\begin{array}{ll}\mathrm{n}^{\circ} & \text { seg. } \\
\mathrm{n}^{\circ} & \text { s.b. } \\
\mathrm{n}^{\prime \prime} & \text { s.s. } \\
& \text { s.s. }\end{array}$ & $\begin{array}{r}54 \\
0 \\
58\end{array}$ & $\begin{array}{r}60 \\
1 \\
64\end{array}$ & $\begin{array}{r}62 \\
0 \\
71\end{array}$ & $\begin{array}{r}64 \\
0 \\
76\end{array}$ & $\begin{array}{r}67 \\
0 \\
76\end{array}$ & $\begin{array}{r}68 \\
0 \\
82\end{array}$ & $\begin{array}{r}68 \\
9 \\
64\end{array}$ & $\begin{array}{r}69 \\
0 \\
80\end{array}$ & $\begin{array}{r}70 \\
0 \\
82\end{array}$ & $\begin{array}{r}71 \\
0 \\
77\end{array}$ & $\begin{array}{r}73 \\
0 \\
78\end{array}$ & & & \\
\hline s.s. + s.b. & 100 & 98 & 100 & 100 & 100 & 100 & 88 & 100 & 100 & 100 & 100 & & & \\
\hline
\end{tabular}

\section{3. - RHYACODRILUS SUBTERRANEUS HRABE, 1963}

En un trabajo precedente (Martinez-Ansemil y Giani, 1980) hemos señalado la presencia de $R$. subterraneus en la Península Ibérica (río Tambre); esta especie sólo había sido capturada hasta entonces en su

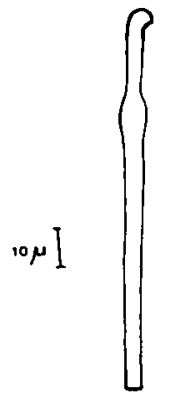

FIG. 2. - Rhyacodrilus subterraneus: seda penial.

localidad tipo en Alemania del Este. Un detenido examen del único individuo que habíamos encontrado en estado de madurez sexual 
(fecha de captura: 5-6-79), nos permite ahora completar la descripción de sus sedas peniales, de las que Hrabe (1963) solo había podido observar su extremo distal.

Diagnosis

$96 \mu$ de longitud y $4,5 \mu$ de diámetro. Son sedas con punta simple y roma, en las que se observa la prensencia de un nódulo a $21 \mu$ de su extremo distal (fig. 2).

\section{4. - TUBIFICIDAE GEN. SP.}

\subsection{Material examinado - hábitat}

Tubificidae gen. sp. es, muy probablemente, una nueva especie de Tubificidae de la que, desafortunadamente, no hemos podido capturar todavía ningún individuo con el aparato genital completamente desarrollado. El número total de individuos encontrados hasta la fecha se eleva a 6. Todos ellos fueron hallados en un estado de desarrollo bastante avanzado el 10-6-79 en la estación de Ponte Maceira en un fondo de cantos rodados bajo una corriente lenta.

\subsection{Diagnosis}

A la espera de la captura de individuos sexualmente desarrollados que nos permitan realizar un encuadramiento taxonómico preciso de esta especie, damos a continuación una descripción de las caracterís. ticas que hemos podido observar en los seis especímenes capturados:

Cuerpo largo y muy delgado: 77 segmentos, $18 \mathrm{~mm}$. de longitud y de 270 a $300 \mu$ de diámetro máximo en el individuo más desarrollado (fijado en formol).

Doble segmentación a partir' del metámero II. Prostomio cónico.

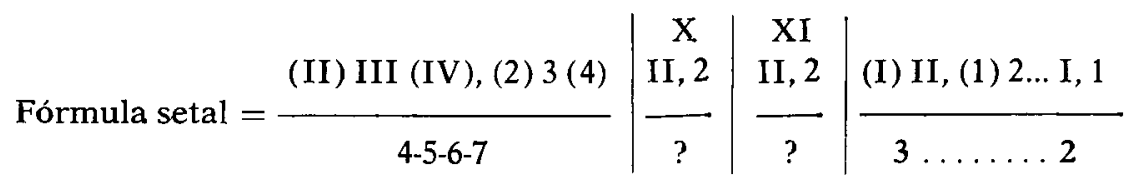

Haces dorsales constituidos por sedas capilares con denticulaciones laterales y sedas pectinadas con los dientes externos de igual longitud y dos finos dientes intermedios (fig. 3 B). En la región anterior del cuerpo se encuentran de 2 a 4 sedas capilares y de 2 a 4 sedas pectinadas por cada haz; la longitud de las capilares varía entre 150 y $300 \mu$, aumentando progresivamente hasta los metámeros VII u VIII y disminuyendo a continuación y la longitud de las pectinadas oscila entre 
38 y $55 \mu$. En la región media, cada haz está formado por 1 o 2 sedas capilares y 1 o 2 sedas pectinadas; los haces de la región posterior solo tienen una seda de cada tipo. En estas dos últimas regiones, la longitud de las sedas capilares varía entre 100 y $135 \mu$ y la de las sedas pectinadas se sitúa alrededor de las $35 \mu$.

Sedas ventrales de $50 \mu$ de longitud aproximadamente ; nódulo distal. De 4 a 7 por haz en la región preclitelar, 3 en la región media y 2 en la región posterior del cuerpo. Se trata, en su mayor parte, de sedas bífidas, con los dientes iguales y poco divergentes, pero en los haces
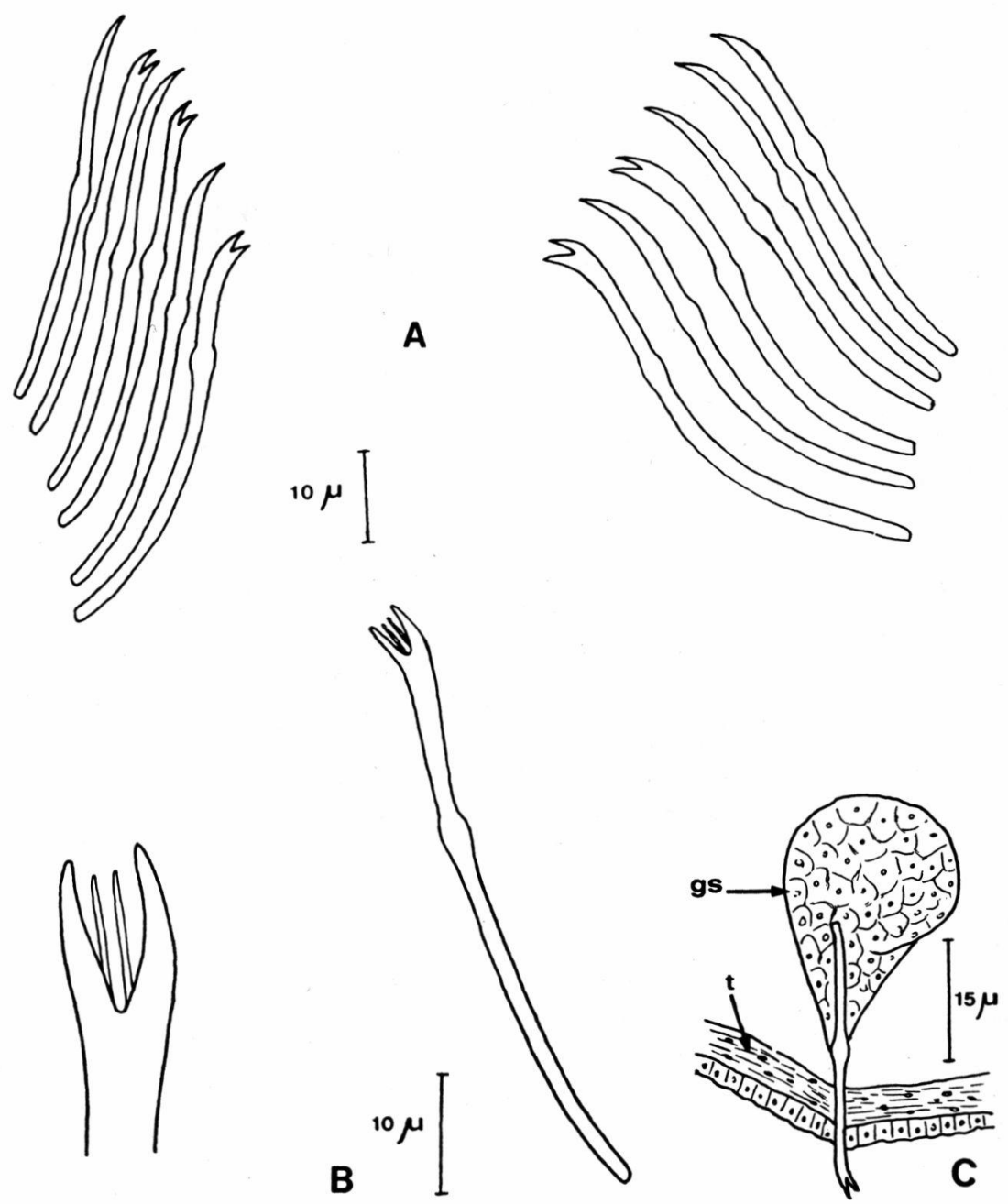

Fig. 3. - Tubificidae gen. sp. : A. dos haces de sedas ventrales de la región anterior; B. seda dorsal pectinada ; C. implantación de una seda ventral : gs : glándula setal, $\mathrm{t}$ : tegumento. 
de los metámeros II a VIII se encuentran además un cierto número de sedas con punta simple (tabla II y fig. $3 \mathrm{~A}$ ).

Tabla II. - Número de sedas con punta simple ( $n^{\circ}$ s.s.) y de sedas con punta bífida $\left(n^{\circ}\right.$ s.b.) en los haces ventrales del especimen más desarrollo de Tubificidac gen. sp.

\begin{tabular}{llcccccccc}
\hline Segmentos & & II & III & IV & V & VI & VII & VIII & IX \\
\hline Lado ventral & $\mathrm{n}^{\circ}$ s.s. & 3 & 3 & 4 & 3 & 1 & 1 & 1 & 0 \\
derecho & $\mathrm{n}^{\circ}$ s.b. & 2 & 3 & 3 & 4 & 6 & 6 & 4 & 5 \\
Lado ventral & $\mathrm{n}^{\circ}$ s.s. & 3 & 4 & 4 & 3 & 0 & 1 & 1 & 0 \\
izquierdo & $\mathrm{n}^{\circ}$ s.b. & 2 & 2 & 3 & 3 & 7 & 6 & 4 & 5 \\
\hline
\end{tabular}

No hemos constatado la presencia de sedas ventrales en los segmentos X y XI de los individuos más desarrollados.

En la base de cada haz de sedas ventrales se encuentra una glándula setal granulosa netamente diferenciada y claramente visible al microscopio óptico incluso en los montajes in toto no aclarados (fig. $3 \mathrm{C}$ ).

Ausencia de celomocitos en la cavidad celómica.

Presencia de glándulas faríngeas en los segmentos IV, V y VI.

\section{TRABAJOS CITADOS}

Brinkhurst (R. O.) y JAmieson (B.G.M.). 1971. - Aquatic Oligochaeta of the World. Oliver and Boyd, Edinburgh, $860 \mathrm{p}$.

Ersteus (C.). 1979 a. - Taxonomic revision of the marine genus Phallodrilus Pierantoni (Oligochaeta, Tubificidae) with descriptions of thirteen new species. Zoologica Scripta, $8:$ 187-208.

ERsÉus (C.). 1979 b. - Taxonomic revision of the marine genera Bathydrilus Cook and Macroseta Erséus (Oligochaeta, Tubificidae) with descriptions of six new species and subspecies. Zoologica Scripta, $8: 139-151$.

ERSEUUS (C.). 1981. - Taxonomic studies of Phallodrilinae (Oligochaeta, Tubificidae) from the Great Barrier Reef and the Comoro Islands with descriptions of ten new species and one new genus. Zoologica Scripta, $10: 15-31$.

Hrabe (S.). 1963. - Rhyacodrilus subterraneus n. sp., eine neue Tubificiden Art aus den Brunnen in der Umgebung von Leipzig. Zoot. Anz., 170: 249-252.

Hrabe (S.). 1964. - On Peloscolex svirenkoi (Jarosenko) and some other species of the genus Peloscolex. Spisy. Prir. Fak. Univ. J.E. Purkyne, Brne, 450 : 101-112.

Hrabe (S.). 1973. - On a collection of Oligochaeta from various part of Yugoslavia. Biol. Vest., 21 : $39-50$.

Martinez-AnSemil (E.). 1981. - Estudio taxonómico y ecológico comparativo de los Oligoquetos de los ríos Tambre (Galicia) y Argens (Sur de Francia). Tesis, Santiago de Compostela, 358 p.

Martinez-Ansemil (E.) y Giani (N.). 1980. - Premières données sur les Oligo chètes aquatiques de la Péninsule Ibérique. Annls Limnol., 16 (1):43-54.

Piguet (E.) y Bretscher (K.). 1913. - Oligochètes. Catalogue des Invertébrés de la Suisse. Mus. Hist. Nat. Genève, 7 : 1-214.

RandolPh (H.). 1892. - Beitrag zur Kenntnis der Tubificiden. Jena z. Naturw., 27 : 463-476. 